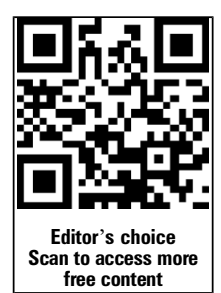
free content

${ }^{1}$ Department of

Ophthalmology, College

of Medicine, Pusan National

University, Yangsan,

Republic of Korea

${ }^{2}$ Medical Research Institute,

Pusan National University

Hospital, Busan,

Republic of Korea

${ }^{3}$ Department of

Ophthalmology, Gyeongsang

National University, JinJu,

Republic of Korea

${ }^{4}$ Department of

Ophthalmology, Haeundae Paik Hospital, College of Medicine,

Inje University, Busan,

Republic of Korea

${ }^{5}$ Department of

Ophthalmology, Busan Paik

Hospital, College of Medicine,

Inje University, Busan,

Republic of Korea

${ }^{6}$ Department of

Ophthalmology, Gospel

hospital, College of Medicine,

Kosin University, Busan,

Republic of Korea

${ }^{7}$ Institute for medicine, Kosin

University, Busan, Republic of Korea

${ }^{8}$ Research Institute for

Convergence of Biomedical

Science and Technology,

Pusan National University

Yangsan Hospital, Yangsan,

Republic of Korea

\section{Correspondence to}

Professor Ji Eun Lee,

Department of Ophthalmology,

Pusan National University

Hospital, 179 Gudeok-ro,

Seo-gu, Busan 602-739,

Republic of Korea;

jlee@pusan.ac.kr

Received 24 January 2015 Revised 4 August 2015

Accepted 6 August 2015

Published Online First

31 August 2015

CrossMark

To cite: Lee JE, Shin MK,

Chung IY, et al. Br J

Ophthalmol 2016;100:

652-659.

\title{
Topographical relationship between the choroidal watershed zone and submacular idiopathic choroidal neovascularisation
}

\author{
Ji Eun Lee, ${ }^{1,2}$ Min Kyu Shin, ${ }^{1,2}$ In Young Chung, ${ }^{3}$ Joo Eun Lee, ${ }^{4}$ Hyun Woong Kim, ${ }^{5}$ \\ Sang Joon Lee, ${ }^{6,7}$ Sung Who Park, ${ }^{1,2}$ Ik Soo Byon ${ }^{1,8}$
}

\begin{abstract}
Aims To investigate the relationship between idiopathic choroidal neovascularisation (CNV) and choroidal watershed zones (CWZs) using indocyanine green angiography (ICGA).
\end{abstract}

Design Multicentre, retrospective, interventional case series.

Methods The medical records and ICGA findings of 44 patients (44 eyes) diagnosed with idiopathic CNV were reviewed. CWZs, defined as hypofluorescence that disappeared during the early phase of ICGA, were classified, and the findings were compared with those of a control group of 30 eyes. The topographical relationship between CWZs and CNV was evaluated. Visual acuity and recurrence were analysed according to the CWZ classification.

Results The CNV lesion was subfoveal in 16 eyes, juxtafoveal in 12 eyes and extrafoveal in 16 eyes. The most common types of CWZs were stellate (23 eyes, $52.3 \%$ ) and vertical (19 eyes, 43.2\%). CWZs involving the fovea were seen in more patients with idiopathic CNV (37 eyes, 84.1\%) than in the control group (11 eyes, 36.7\%, $p<0.001$ ). The topographical relationship between CWZs and CNV was determined in 42 eyes $(95.5 \%)$, with the CNV located within the CWZ in 39 eyes and at the margin in 3 eyes. Extrafoveal CNV was within the CWZ in all 16 affected eyes. At 6 months, visual acuity was significantly worse in patients with subfoveal CNV ( $p=0.028)$ or stellate CWZs ( $p=0.039$ ).

Conclusions The findings of a CWZ were related to the location and functional outcome of idiopathic CNV. Our results suggest that choroidal circulation is a predisposing factor for the development of CNV in young patients.

\section{INTRODUCTION}

Choroidal neovascularisation (CNV) may be complicated by various conditions, including age-related macular degeneration (AMD), white dot syndromes, high myopia, presumed ocular histoplasmosis syndrome, angioid streak and traumatic choroidal rupture. Idiopathic $\mathrm{CNV}$ refers to $\mathrm{CNV}$ that develops without any predisposing abnormalities, ${ }^{1-3}$ and few studies have suggested mechanisms for idiopathic CNV. Yang et $a l^{4}$ reported that inflammatory cytokines such as vascular endothelial growth factor (VEGF) and immunoglobulin $\mathrm{E}$ were detected at high levels in the serum of patients with idiopathic CNV. Favourable outcomes of anti-VEGF treatments ${ }^{5-8}$ support this theory. However, no clinical findings have been reported suggesting a possible source of VEGF.

The results of several studies have demonstrated that the human choroid has endarterial systems supplied by various posterior ciliary arteries (PCAs) and that multiple watershed zones may be located in the macula (figure 1). ${ }^{9}{ }^{10}$ A choroidal watershed zone (CWZ) was demonstrated by fluorescein angiography (FA) to course vertically around the optic nerve head $(\mathrm{ONH})$ typically in normal subjects, ${ }^{11}$ and shows various patterns with indocyanine green angiography (ICGA). ${ }^{12}$ The CWZ has a topographical relationship with CNV in exudative AMD and was proposed to be related to the development of $\mathrm{CNV}$ with an ischaemic mechanism. ${ }^{13-15}$

The purpose of this study was to investigate the topographical relationship of CWZs and idiopathic $\mathrm{CNV}$ with the objective of suggesting a mechanism for idiopathic $\mathrm{CNV}$ development.

\section{METHODS}

The medical records of patients who were diagnosed with idiopathic CNV in five hospitals (Pusan National University Hospital, Gyeongsang National University Hospital, Haeundae Paik Hospital, Busan Paik Hospital and Gospel Hospital) from 2008 to 2013 were reviewed retrospectively. The control cohort, using the same inclusion criteria excluding the presence of idiopathic CNV, was collected in Pusan National University Hospital in 2014. The study conducted adhered to the tenets of the Declaration of Helsinki.

The presence of $\mathrm{CNV}$ was confirmed using FA and optical coherence tomography (OCT). The inclusion criteria were as follows: age $\leq 45$ years, an axial length of $<26 \mathrm{~mm}$ and a spherical equivalent refractive error of not more than -5.0 dioptres (D). Patients with polypoidal choroidal vasculopathy, white dot syndromes, presumed ocular histoplasmosis syndrome, high myopia, hereditary dystrophy, angioid streak and other choroidal abnormalities related to CNV were excluded. Eyes with possible predisposing factors such as a choroidal scar, soft drusen, and a history of uveitis or central serous chorioretinopathy were also excluded.

Patients were evaluated using slit-lamp biomicroscopy with a $90 \mathrm{D}$ lens, fundus photography and OCT (Cirrus HD-OCT; Carl Zeiss Meditec, Dublin, California, USA). Visual acuity was measured using the Snellen chart and converted to the logarithm of the minimum angle of resolution. FA and ICGA were performed using a confocal scanning laser ophthalmoscope (Heidelberg Retina 

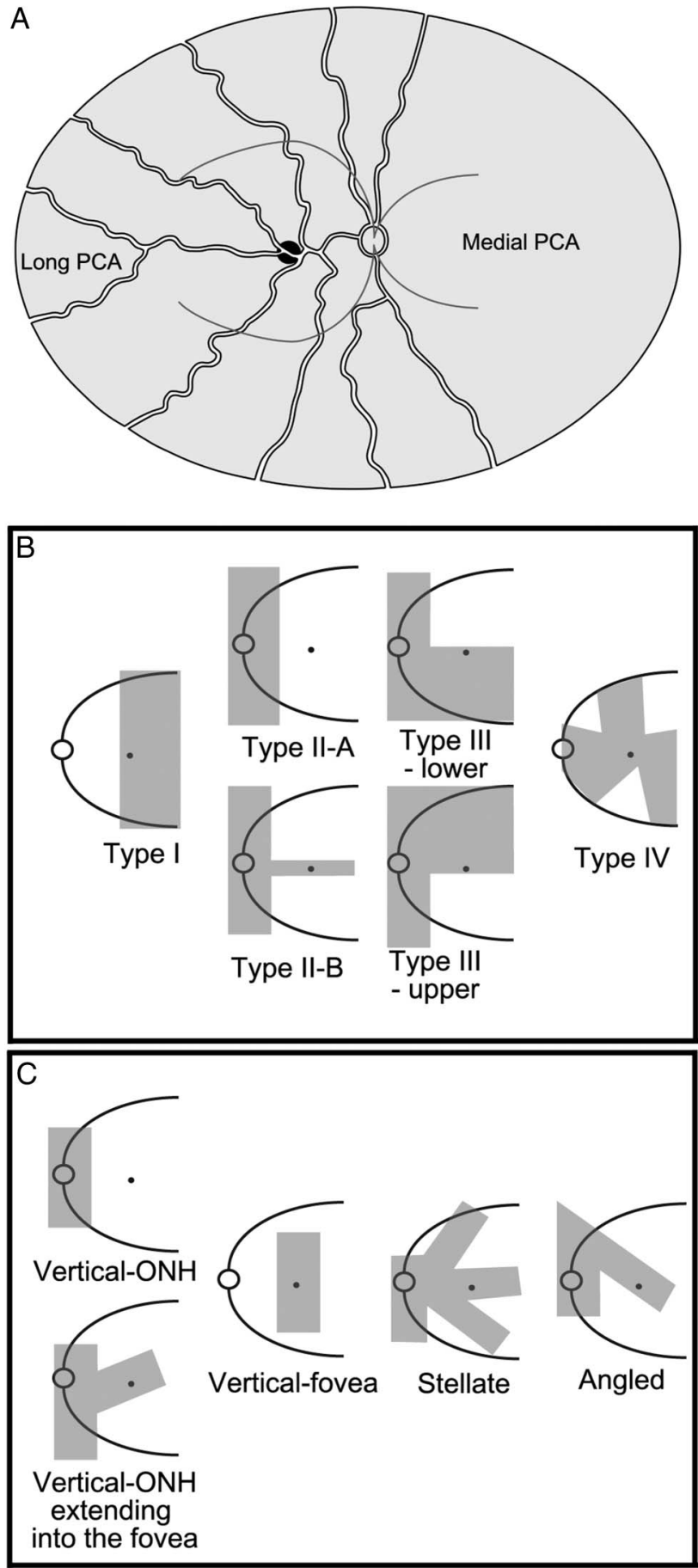

Figure 1 Segmental distribution of the human choroid by the various short posterior ciliary arteries (A). ${ }^{14}$ The choroidal watershed zones are shown with indocyanine green angiography according to the classifications of Hayashi and de Laey as well as Mendrinos and Pournaras (B and C). ${ }^{10}{ }^{11} \mathrm{PCA}$, posterior ciliary artery; $\mathrm{ONH}$, optic nerve head.

Angiograph 2; Heidelberg Engineering, Heidelberg, Germany). For the early phase, the images were immediately obtained after injection of fluorescein or indocyanine green dye at a rate of at least two to three images per second for $30 \mathrm{~s}$.

A CWZ was defined as delayed filling of the choriocapillaris which appeared as a hypofluorescence and then disappeared during the early phase of FA and ICGA. ${ }^{10}{ }^{12-15}$ However, since assessment in FA was unreliable due to the pigmentation in
Asians, CWZ was evaluated in ICGA only. ICGA findings were analysed independently by two readers (JEL and MKS), and the extent of their agreement was calculated. For discrepant assessments, the final decision was made on the basis of a consensus among the two readers.

CWZs were categorised using two classification systems based on prior reports. Hayashi and de Laey ${ }^{12}$ classified CWZs into four categories: I, nasal filling; II-A, temporal filling; II-B, temporal filling with delayed fluorescence in the foveal area; III, upper or lower temporal quadrant filling; and IV, scattered dye filling (figure 1B). Mendrinos and Pournaras ${ }^{13}$ classified CWZs into three categories: stellate, vertical and angled. Vertical CWZs were subgrouped as follows: coursing through the $\mathrm{ONH}$ and extending to the fovea; coursing through the $\mathrm{ONH}$ without extending to the fovea; and coursing through the fovea without extending to the $\mathrm{ONH}$ (figure 1C).

Location of CNV was categorised as subfoveal, juxtafoveal and extrafoveal, based on distance of $200 \mu \mathrm{m}$ from the foveal centre for consistency with AMD. ${ }^{16}$ The topographical relationship between a CWZ and an area of CNV was analysed as 'within CWZ', 'at the margin of the CWZ' or 'out of the CWZ'. ${ }^{13}$ An isolated area of delayed filling besides the abovementioned CWZ categories were analysed separately. Foveal involvement of a CWZ was also analysed.

ICGA findings in the idiopathic CNV group and the control group were compared. The visual acuity, treatments, recurrence and treatment outcomes were evaluated in patients who had been followed up for 6 months or more to determine correlations with the CWZ and CNV.

Categorical variables were compared by $\chi^{2}$ test or Fisher's exact test. Visual acuity was compared using non-parametrical tests (Mann-Whitney U test, Wilcoxon signed-rank test and Kruskal-Wallis test). Statistical analysis was performed using IBM SPSS Statistics V.21 (International Business Machines Corporation, Armonk, New York, USA).

\section{RESULTS}

Fifty-six eyes of 56 consecutive patients were reviewed, and 12 eyes were excluded because of poor-quality ICGA images or possible correlations with other predisposing conditions listed in the exclusion criteria. Thus, 44 eyes were included in this study, and their baseline profiles are summarised in table 1 . The average age was 33.7 years (range $18-45$ years at diagnosis).

Table 1 Baseline characteristics of patients with idiopathic choroidal neovascularisation (CNV) and the control group

\begin{tabular}{lccc}
\hline Parameters & Idiopathic CNV & Control & p Value \\
\hline $\begin{array}{l}\text { Number } \\
\text { Gender }\end{array}$ & 44 & 30 & \\
$\quad$ Male, N (\%) & $15(34.1)$ & $13(43.3)$ & $0.421^{*}$ \\
$\quad$ Female, N (\%) & $29(65.9)$ & $17(56.7)$ & \\
Mean age, years (range) & $33.7(18-45)$ & $30.6(22-44)$ & $0.064 \dagger$ \\
Mean refractive error, & $-2.01(+1.75--5.0)$ & $-1.45(+0.83--4.33)$ & $0.179 \dagger$ \\
dioptre (range) & & & \\
Location & & & \\
$\quad$ Subfoveal, N (\%) & $16(36.4)$ & NA§ & \\
Juxtafoveal, N (\%) & $12(27.3)$ & & \\
Extrafoveal, N (\%) & $16(36.4)$ & & \\
\hline * $\chi^{2}$ test. \\
†Mann-Whitney U test. \\
CNV, choroidal neovascularisation; NA, not applicable.
\end{tabular}


Table 2 Classification of a choroidal watershed zone in patients with idiopathic choroidal neovascularisation (CNV) and normal controls

\begin{tabular}{lccc}
\hline & \multicolumn{1}{c}{ Idiopathic } & & \\
& CNV & Control & p Value* \\
\hline Hayashi's classification & & & \\
$\quad$ Type II-A & $7(15.9 \%)$ & $7(23.3 \%)$ & 0.261 \\
Type II-B & $5(11.4 \%)$ & $0(0 \%)$ & \\
Type III & $9(20.5 \%)$ & $7(23.3 \%)$ & \\
$\quad$ Type IV & $23(52.3 \%)$ & $16(53.3 \%)$ & \\
Mendrinos' classification & & & \\
$\quad$ Stellate & $23(52.3 \%)$ & $15(50.0 \%)$ & 0.150 \\
$\quad$ Vertical & $19(43.2 \%)$ & $15(50.0 \%)$ & \\
$\quad$ Extending to the fovea & $11(57.9 \%)$ & $3(10.0 \%)$ & \\
$\quad$ Without extending to the fovea & $7(36.8 \%)$ & $11(36.7 \%)$ & \\
$\quad$ Through the fovea & $1(5.3 \%)$ & $1(3.3 \%)$ & \\
$\quad$ Angled & $2(4.5 \%)$ & $0(0 \%)$ & \\
Foveal involvement & & & \\
$\quad$ Foveal & $37(84.1 \%)$ & $11(36.7 \%)$ & $<0.0001$ \\
$\quad$ Non-foveal & $7(15.9 \%)$ & $19(63.3 \%)$ & \\
\hline${ }^{*} \chi^{2}$ test. & & & \\
CNV, choroidal neovascularisation. & & & \\
\end{tabular}

The control group was composed of a cohort of 30 normal controls (20-44 years old), who showed no significant difference from the idiopathic CNV group in sex, age and refractive error.

\section{Classification of CWZs}

A CWZ began to show just after appearance of choroidal artery between $6 \mathrm{~s}$ and $16 \mathrm{~s}$ after injection of indocyanine green solution, depending on the injection site. A CWZ was discernible for $5.9 \mathrm{~s}( \pm 1.8$, range $3-12 \mathrm{~s})$ in idiopathic CNV group, and $5.1 \mathrm{~s}( \pm 1.3$, range $3-8 \mathrm{~s})$ in control group. The difference was not statistically significant ( $\mathrm{p}=0.071$, Mann-Whitney U test).

The agreement was $77.3 \%$ for Mendrinos' classification, $73.3 \%$ for Hayashi's classification and $90.7 \%$ for foveal involvement of a CWZ. Table 2 shows the results of the CWZ classification in ICGA. According to Hayashi's classification, type IV was most common (23 eyes, 52.3\%). Type II-A was seen in seven eyes (15.9\%), type II-B in five eyes (11.4\%) and type III in nine eyes (20.5\%). Using Mendrinos' classification, stellate CWZs were most common and were found in 23 eyes $(52.3 \%$, figure 2). Vertically oriented CWZs (figures 3-6) were seen in 19 eyes (43.2\%) and angled CWZs in 2 eyes (4.5\%). In the control group, the distribution did not differ from that of the patients with idiopathic $\mathrm{CNV}$ in both $\mathrm{CWZ}$ classifications ( $p=0.150$ and $0.261, \chi^{2}$ test).

CWZs involving the fovea were seen in more patients with idiopathic CNV (37 eyes, 84.1\%) than in the control group (11 eyes, $36.7 \%, \mathrm{p}<0.001)$. The foveal area in the control group was commonly spared even in eyes with stellate CWZs (7 of 13 eyes, figure 7). In addition to the abovementioned CWZ classifications, 11 eyes $(25.0 \%)$ had isolated areas of delayed filling (figures 5 and 6). Some of these areas appeared to correlate with the distribution of short PCAs rather than with the CWZ (figure 5).
Figure 2 Subfoveal idiopathic choroidal neovascularisation (CNV) in an 18-year-old woman (A). Optical coherence tomography depicts CNV and subretinal fluid (B). During the early phase of fluorescein angiography (C) and indocyanine green angiography (D), CNV located at the margin of a stellate choroidal watershed zone (CWZ), or type IV according to Hayashi's classification, is shown. The CWZ is not seen during the late phase of fluorescein angiography (E).

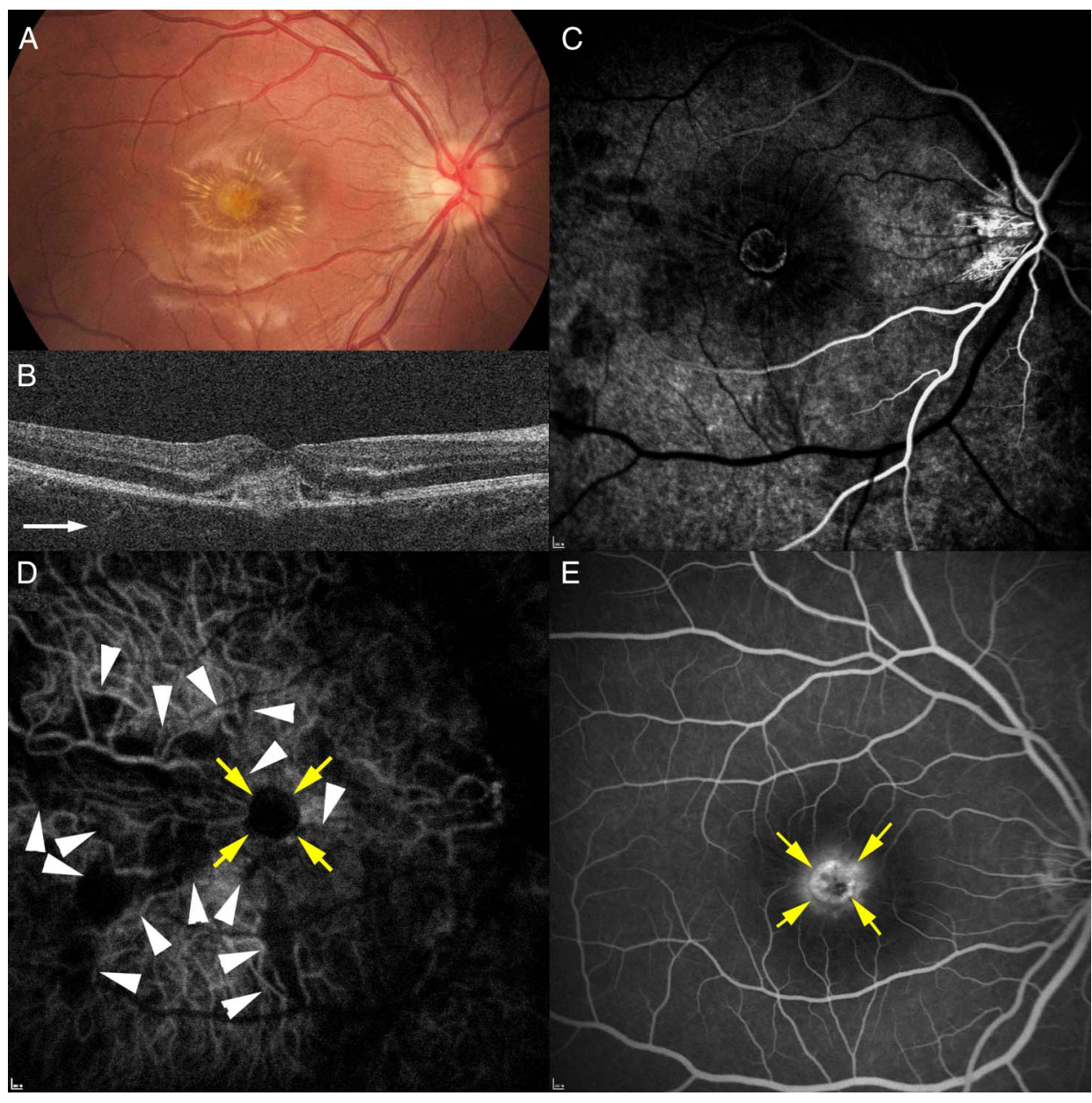

Lee JE, et al. Br J Ophthalmol 2016;100:652-659. doi:10.1136/bjophthalmol-2015-306678 
Figure 3 Extrafoveal idiopathic choroidal neovascularisation (CNV) in a 36-year-old woman (A). CNV and macular oedema are seen in optical coherence tomography (B). Early-phase fluorescein angiography $(C)$ and indocyanine green angiography (D) reveal a choroidal watershed zone (CWZ, arrowheads) coursing through the optic nerve head and extending to the fovea. It was classified as Hayashi type II-B. Extrafoveal CNV (yellow arrows) was located within the CWZ (D and $E$ ).

Figure 4 Extrafoveal choroidal neovascularisation (CNV) developed in a 42-year-old man (A). CNV and subfoveal fluid are demonstrated in optical coherence tomography (B). Fluorescein angiography (C) and indocyanine green angiography (D) show a vertical choroidal watershed zone (CWZ) coursing through the fovea during the early phase (arrowheads). CNV (yellow arrows) was located in the CWZ (D and E).
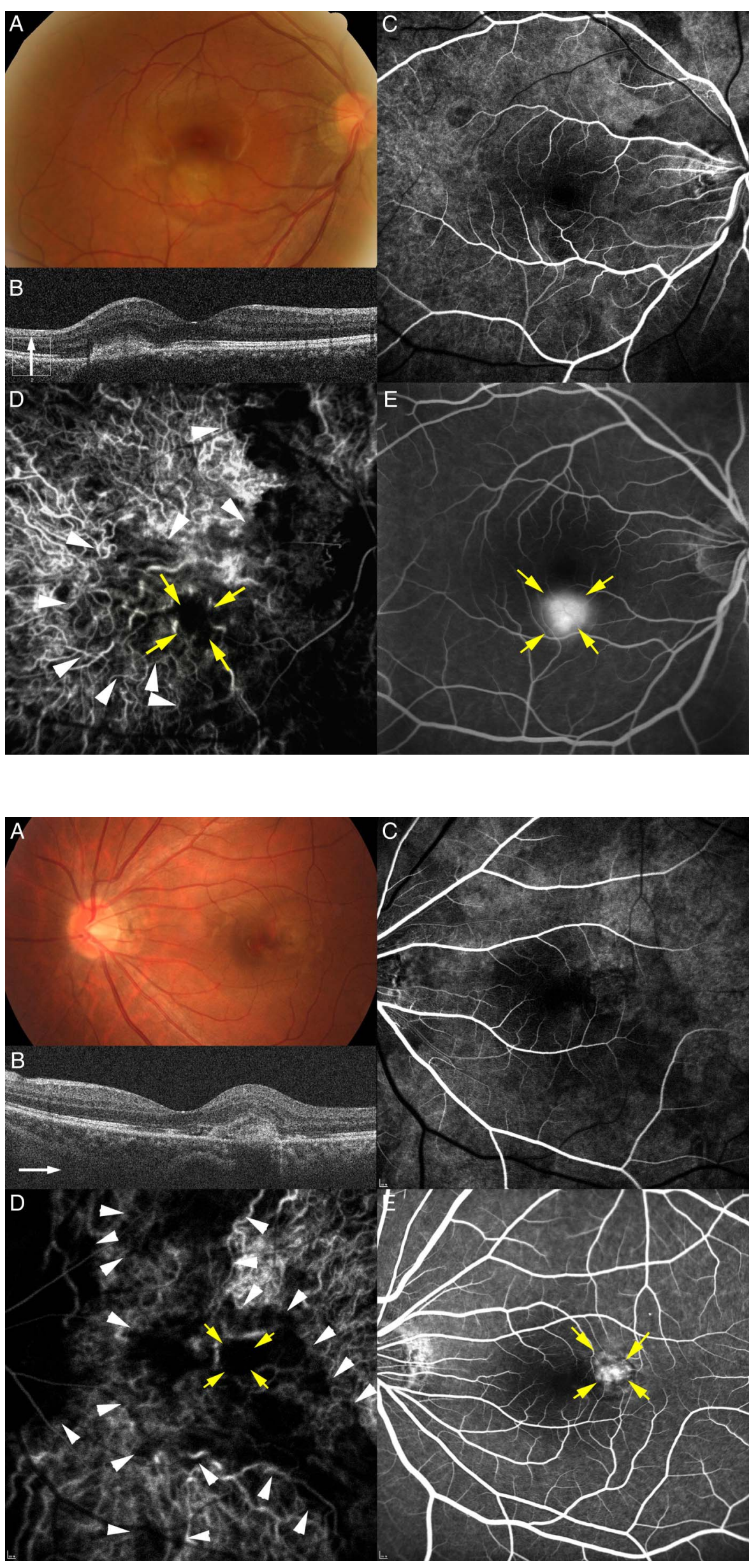


\section{Topographical relationship between CWZ and CNV}

CNV location was subfoveal in 16 eyes (36.4\%), juxtafoveal in 12 eyes $(27.3 \%)$ and extrafoveal in 16 eyes (36.4\%, table 1 ). CNV was located within an area of delayed filling in 39 eyes $(88.6 \%$, figures $3-5)$ and at the margin in 3 eyes $(6.8 \%$, figure 2). Two eyes (4.5\%), one with subfoveal CNV and the other with juxtafoveal CNV, had CNV outside the areas of a vertical CWZ coursing through the disc (figure 6). Extrafoveal $\mathrm{CNV}$ was located within an area of delayed filling in all eyes (figures 3-5). Six eyes (13.6\%) had a CNV located within the isolated areas of delayed filling (figure 5).

No relationship was found between CNV location and CWZ classification $\left(p=0.342-0.510, \chi^{2}\right.$ test, table 3$)$.

\section{Treatment outcomes}

The primary treatment was intravitreal ranibizumab for 10 patients, intravitreal bevacizumab for 31 patients and photodynamic therapy for 1 patient. Two patients received no treatment. Thirty-six patients were followed up for $\geq 6$ months; 19 patients were followed up for $\geq 12$ months. The mean follow-up duration was 16.0 months.

In patients followed up for $\geq 6$ months, the median visual acuity significantly improved from 20/60 at baseline to 20/20 at 6 months $(p=0.002)$ and $20 / 25$ at 12 months $(p=0.003$, Wilcoxon signed-rank test). Patients received photodynamic therapy or an intravitreal injection 2.7 times by 6 months and 3.1 times by 1 year in average. Better baseline visual acuity was related to better visual outcomes $(p<0.001)$. At 6 months, visual acuity was better for the eyes with non-subfoveal CNV than for the eyes with subfoveal CNV ( $p=0.028$, Mann-Whitney U test, table 4). A stellate CWZ or type IV CWZ was related to significantly worse visual acuity than the other types $(p=0.039$, Mann-Whitney $U$ test, table 4$)$. No other correlation was found $(p=0.058-0.746$, Mann-Whitney U test and Kruskal-Wallis test).

There was a recurrence of CNV activity in 8 of the 36 patients $(22.2 \%)$ by 6 months and in 7 of the 19 patients (36.8\%) by 12 months. A vertical CWZ extending to the fovea was the most common type among the patients showing recurrence by 6 months (4 eyes, 50.0\%) and 12 months (5 eyes, $71.4 \%$ ), but recurrence was not statistically significant with regards to classification of CWZ ( $p=0.334-0.694$, Fisher's exact test and $\chi^{2}$ test).

\section{DISCUSSION}

Our study revealed that idiopathic CNV had topographical relationships with CWZs, suggesting that delayed perfusion of the choriocapillaris may predispose patients to idiopathic $\mathrm{CNV}$ development. Hayreh suggested a pathological role of a CWZ in development of CNV for the first time in 1974. ${ }^{17}$ However, because of the unique features of the choroidal vasculature the debate regarding a hypoxic role for CWZs continues. For example, angiography in vivo shows that the choroidal lobule is supplied by a terminal arteriole not anastomosing with the adjacent lobule ${ }^{18}$ but a corrosion cast study demonstrated that the submacular choroidal vessels anastomose at various levels of capillaries, arterioles and venules. ${ }^{19}$
Figure 5 A segmental distribution of a short posterior artery is marked as the dark area in the segmented choroidal perfusion diagram (A). Fluorescein angiography (B) and indocyanine green angiography (C, arrowheads) in a 30-year-old woman demonstrated delayed choroidal filling, which correlates with the segment marked in the diagram. Extrafoveal idiopathic choroidal neovascularisation (CNV) developed within the area of delayed filling (C and D, yellow arrows). (E) Optical coherence tomography shows CNV developed in the extrafoveal region, macular oedema and subretinal fluid involving the fovea.

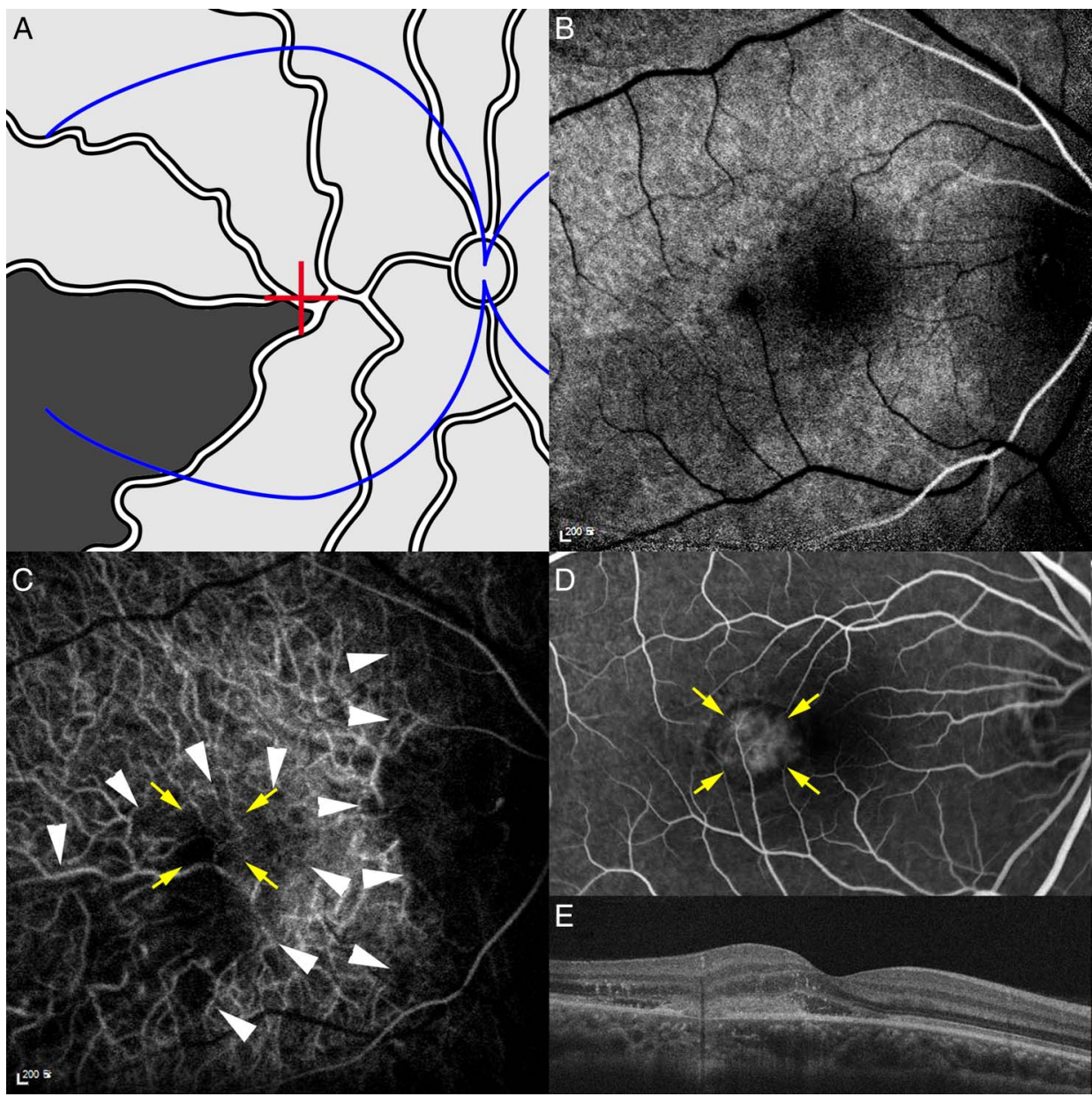

Lee JE, et al. Br J Ophthalmol 2016;100:652-659. doi:10.1136/bjophthalmol-2015-306678 
Figure 6 Juxtafoveal idiopathic choroidal neovascularisation (CNV) in a 39-year-old man (A). Optical coherence tomography shows CNV (B). Choroidal watershed zones (CWZs) were less distinctly demonstrated with fluorescein angiography (C) than indocyanine green angiography (D), where an isolated area of delayed filling was seen in the macula (arrowheads). Juxtafoveal CNV (yellow arrows) was located outside of the CWZs (D and E).

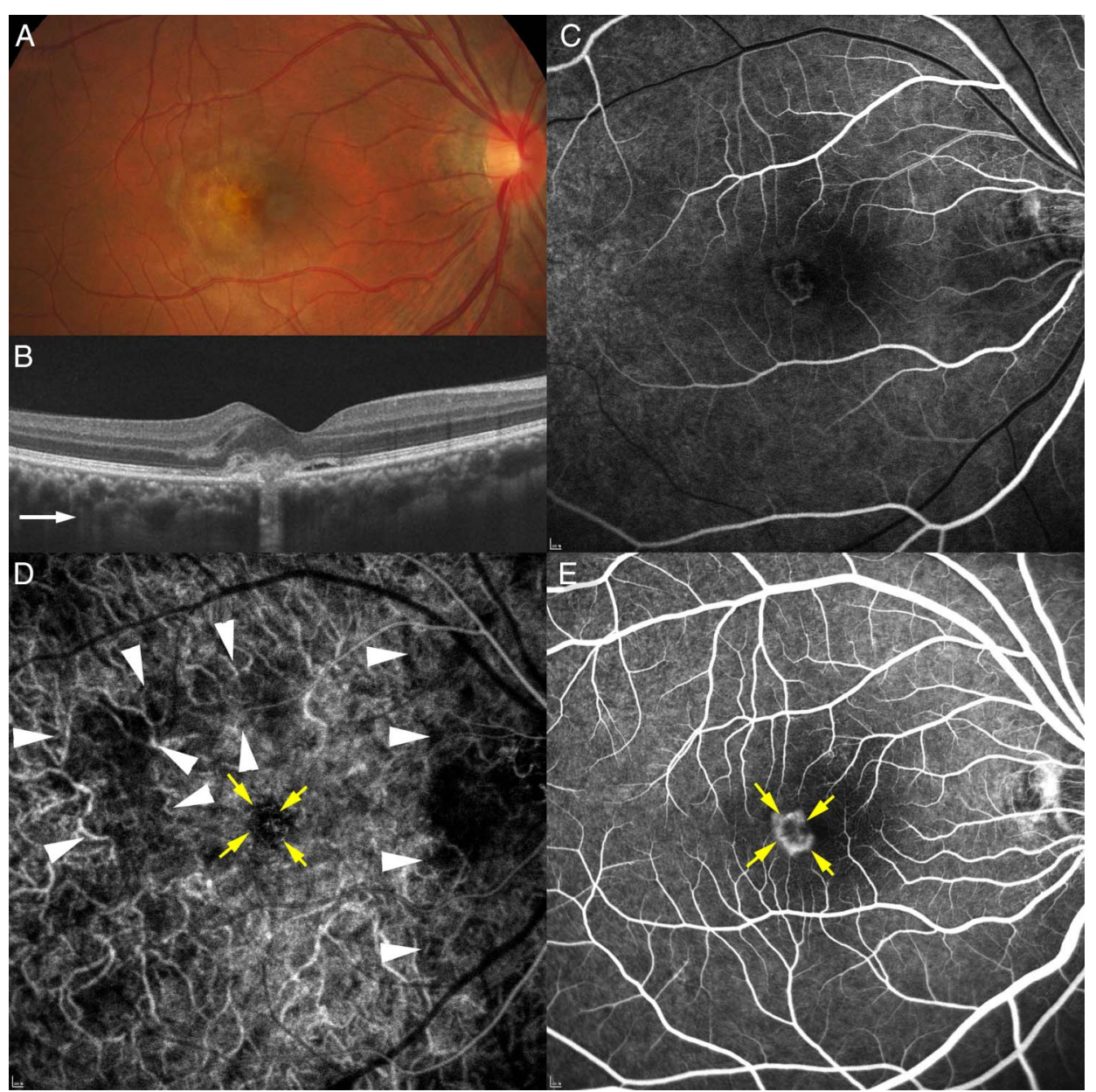

Many studies have substantiated that CWZs play a pathological role. In vivo studies using FA have indicated that the choroid has an endarterial system along with segmental blood flow from the PCAs. ${ }^{9} 17$ Laser-targeted angiography has demonstrated that cross-flow from lobule to lobule does not occur in the normal choroid. ${ }^{20}$ The segmental distribution of choroidal perfusion from the various PCAs has been studied in eyes with ischaemic optic neuropathy or choroidal infarction. ${ }^{21} 22 \mathrm{CWZs}$, representing boundaries situated between the segmental perfusions, ${ }^{10}$ are important in various clinical situations because of their relatively poor blood flow. ${ }^{23}$
Several studies have shown that AMD has a relationship with choroidal filling patterns. ${ }^{13} 1524$ In these studies, the most common CWZ was the stellate type, with a topographical relationship rate from $71 \%$ to $100 \%$. Choroidal thinning and elevated intraocular VEGF levels in patients with AMD also suggest that choroidal ischaemia may lead to $\mathrm{CNV}^{25-27}$ Decreased choroidal circulatory parameters were associated with AMD development and progression. ${ }^{28}$ Moreover, eyes with delayed patchy choroidal filling seen on FA had worse functional outcomes following anti-VEGF treatment. ${ }^{29}$
Figure 7 A stellate choroidal watershed zone is shown (arrowheads) in a 42-year-old patient with idiopathic choroidal neovascularisation (A) and a cohort of 43-years-old normal controls (B). The foveal area is spared in the control.

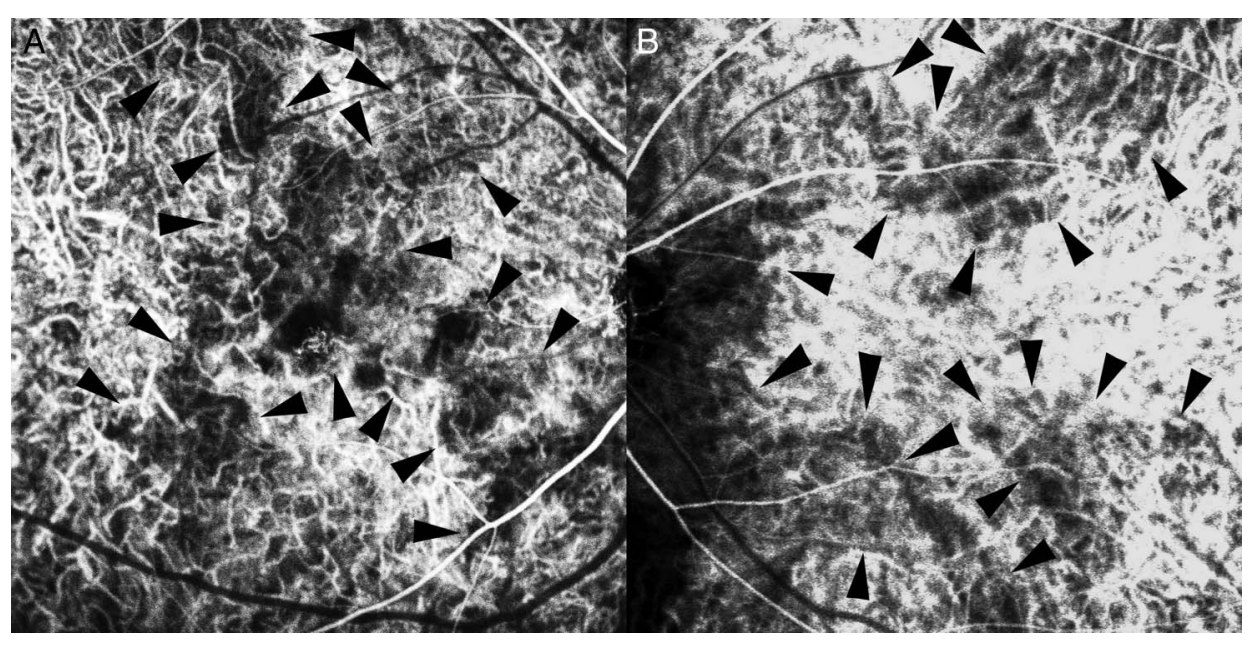


Table 3 Choroidal filling pattern and topographical relationship with idiopathic choroidal neovascularisation (CNV) according to location

\begin{tabular}{|c|c|c|c|c|}
\hline Classification & $\begin{array}{l}\text { Subfoveal } \\
\text { CNV } \\
(\mathrm{N}=16)\end{array}$ & $\begin{array}{l}\text { Juxtafoveal } \\
\text { CNV } \\
(\mathrm{N}=12)\end{array}$ & $\begin{array}{l}\text { Extrafoveal } \\
\text { CNV } \\
(\mathrm{N}=16)\end{array}$ & p Value* \\
\hline \multicolumn{5}{|l|}{ Hayashi's classification } \\
\hline Type II-A & $2(12.5 \%)$ & $2(16.7 \%)$ & $3(18.8 \%)$ & 0.510 \\
\hline Type II-B & $2(12.5 \%)$ & $2(16.7 \%)$ & $1(6.3 \%)$ & \\
\hline Type III & $1(6.3 \%)$ & $4(33.3 \%)$ & $4(25.0 \%)$ & \\
\hline Type IV & $11(68.8 \%)$ & $4(33.3 \%)$ & $8(50.0 \%)$ & \\
\hline \multicolumn{5}{|l|}{ Mendrinos' classification } \\
\hline Stellate & $11(68.8 \%)$ & $4(33.3 \%)$ & $8(50.0 \%)$ & 0.400 \\
\hline Vertical & $5(31.3 \%)$ & $7(58.3 \%)$ & $7(43.8 \%)$ & \\
\hline $\begin{array}{l}\text { Without extending to } \\
\text { the fovea }\end{array}$ & $2(40.0 \%)$ & $2(28.6 \%)$ & $3(42.9 \%)$ & \\
\hline Extending to the fovea & $3(60.0 \%)$ & $4(57.1 \%)$ & $4(57.1 \%)$ & \\
\hline Through the fovea & $0(0.0 \%)$ & $1(14.3 \%)$ & $0(0.0 \%)$ & \\
\hline Angled & $0(0.0 \%)$ & $1(8.3 \%)$ & $1(6.3 \%)$ & \\
\hline \multicolumn{5}{|c|}{ Relationship between CNV and CWZ } \\
\hline Within & $14(75.0 \%)$ & $9(75.0 \%)$ & $16(100 \%)$ & 0.342 \\
\hline At the margin & $1(6.3 \%)$ & $2(16.7 \%)$ & $0(0.0 \%)$ & \\
\hline No relationship & $1(6.3 \%)$ & $1(8.3 \%)$ & $0(0.0 \%)$ & \\
\hline
\end{tabular}

In our study, idiopathic CNV was related to the presence of a CWZ. A stellate or type IV CWZ was found most frequently, concordant with previous reports involving patients with AMD. ${ }^{13} 14$ The topographical relationship between idiopathic $\mathrm{CNV}$ and CWZs was found in 42 eyes (95.5\%). We also found

Table 4 Visual outcomes of idiopathic choroidal neovascularisation (CNV) according to its location and the choroidal watershed zone

\begin{tabular}{|c|c|c|c|}
\hline & \multicolumn{3}{|c|}{ Visual acuity average (range) in logMAR* } \\
\hline & $\begin{array}{l}\text { Baseline } \\
(\mathrm{N}=36)\end{array}$ & $\begin{array}{l}6 \text { months } \\
(\mathrm{N}=36)\end{array}$ & $\begin{array}{l}1 \text { year } \\
(\mathrm{N}=19)\end{array}$ \\
\hline \multicolumn{4}{|l|}{ Hayashi's classification } \\
\hline Type II-A & $0.56(0.0-2.0)$ & $0.08(0.0-0.4)$ & $0.05(0.0-0.1)$ \\
\hline Type II-B & $0.13(0.0-0.3)$ & $0.07(0.0-0.2)$ & $0.03(0.0-0.1)$ \\
\hline Type III & $0.41(0.0-1.4)$ & $0.10(0.0-0.4)$ & $0.14(0.0-0.4)$ \\
\hline Type IV & $0.53(0.0-2.0)$ & $0.30^{*}(0.0-1.7)$ & $0.38(0.0-1.7)$ \\
\hline \multicolumn{4}{|l|}{ Mendrinos' classification } \\
\hline Stellate & $0.53(0.0-2.0)$ & $0.30 *(0.0-1.7)$ & $0.63(0.0-1.7)$ \\
\hline Vertical & $0.39(0.0-2.0)$ & $0.09(0.0-0.4)$ & $0.09(0.0-0.4)$ \\
\hline $\begin{array}{l}\text { Without extending to the } \\
\text { fovea }\end{array}$ & $0.56(0.0-2.0)$ & $0.08(0.0-0.4)$ & $0.05(0.0-0.1)$ \\
\hline Extending to the fovea & $0.18(0.0-0.5)$ & $0.07(0.0-0.4)$ & $0.06(0.0-0.2)$ \\
\hline Through the fovea & 1.40 (NA $\ddagger)$ & 0.40 (NA) & 0.40 (NA) \\
\hline Angled & 0.70 (NA) & 0.00 (NA) & NA (NA) \\
\hline \multicolumn{4}{|l|}{ CNV§ location } \\
\hline Subfoveal & $0.57(0.0-2.0)$ & $0.36 *(0.0-1.7)$ & $0.44(0.0-1.7)$ \\
\hline Juxtafoveal & $0.58(0.2-1.4)$ & $0.11(0.0-0.4)$ & $0.17(0.0-0.4)$ \\
\hline Extrafoveal & $0.30(0.0-1.4)$ & $0.08(0.0-0.6)$ & $0.03(0.0-0.2)$ \\
\hline
\end{tabular}

isolated areas of delayed filling that could not be categorised as typical CWZ in 11 eyes, and 6 of these eyes (54.5\%) had CNV located in those areas. It is noteworthy that extrafoveal CNV was found more frequently in our patients than in those with AMD, and it always had a topographical relationship with a CWZ. These results indicate that the development of idiopathic $\mathrm{CNV}$ is closely associated with delayed choroidal filling, particularly when a CWZ involves the fovea.

The most pathological type of CWZ in our study was the stellate or type IV CWZ, which was associated with significantly worse visual acuity at 6 months follow-up. The stellate shape suggests that multiple CWZs exist among choroidal perfusions, and the choroidal filling is delayed more broadly than the other types. The specific type of CWZ appears to relate more to prognosis than development because, in our study and others, the frequency of a stellate CWZ was only slightly higher (44-60\%) in pathological conditions than in the control groups (3548\%). ${ }^{12} 13$

Subfoveal choroidal thickness was reported thicker in the eye with idiopathic CNV than the fellow eye, and the thickness decreased after anti-VEGF therapy. ${ }^{30}$ Although the choroid is thickest at the fovea and would have high blood flow, these do not necessarily mean that the choroid is non-ischaemic, because larger choroidal vessels occupy most of the thickness and flow. Choroidal thickening would be a sign of congestion of the choroid and stasis in the venous drainage, ${ }^{27}$ and these may cause hypoxia of the choriocapillaris and outer retina.

Choroidal ischaemia would not be the only mechanism for idiopathic CNV. A CWZ was found in all eyes in the control group, and there were two eyes in which CNV developed outside the CWZ. However, none of them was extrafoveal (figure 6). It is known that the fovea is the area most vulnerable to ischaemia, because several sectors of segmental perfusion meet in the foveal area in a wedge shape that forms multiple CWZs (figure 1). ${ }^{17}$ Moreover, the fovea is the most active area demanding oxygen, and predisposing to relative hypoxia than the other area. Accordingly, even when CNV and CWZs are not matched topographically, a possible relationship with the choroidal circulation cannot be excluded.

Our study has several limitations. It was a retrospective study, and the follow-up period was short. Various treatment guidelines and follow-up intervals were applied. OCT images of the choroid were not evaluated using enhanced depth imaging or swept-source OCT. These aspects should be evaluated in future studies.

In conclusion, CWZs were found to be related to idiopathic $\mathrm{CNV}$ topographically, and CWZ classification was a predictor of functional outcomes. These results suggest that choroidal perfusion is an important mechanism predisposing young to middle-aged patients to the development of CNV.

Contributors Study concept and design: JEL; acquisition of data: all authors; analysis and interpretation of data, and preparation of manuscript: JEL, MKS; review of manuscript: all authors. All authors had full access to all the data in the study and take responsibility for the integrity of the data and accuracy of the data analysis.

Competing interests None declared.

Patient consent Obtained.

Ethics approval Institutional Review Board of Pusan National University Hospital. Provenance and peer review Not commissioned; externally peer reviewed.

\section{REFERENCES}

1 Cleasby GW. Idiopathic focal subretinal neovascularization. Am J Ophthalmol 1976;81:590-9. 
2 Ho AC, Yannuzzi LA, Pisicano $K$, et al. The natural history of idiopathic subfoveal choroidal neovascularization. Ophthalmology 1995;102:782-9.

3 Toju R, lida T, Sekiryu T, et al. Near-infrared autofluorescence in patients with idiopathic submacular choroidal neovascularization. Am J Ophthalmol 2012;153:314-19.

4 Yang F, Dou HL, Ma Z, et al. Serum inflammatory factors in patients with idiopathic choroidal neovascularization. Ocul Immunol Inflamm 2010;18:390-4.

5 Zhang $\mathrm{H}$, Liu ZL, Sun $\mathrm{P}$, et al. Intravitreal bevacizumab for treatment of subfoveal idiopathic choroidal neovascularization: results of a 1-year prospective trial. Am J Ophthalmol 2012;153:300-6.

6 Mandal S, Garg S, Venkatesh P, et al. Intravitreal bevacizumab for subfoveal idiopathic choroidal neovascularization. Arch Ophthalmol 2007;125:1487-92.

7 Kang HM, Koh HJ. Intravitreal anti-vascular endothelial growth factor therapy versus photodynamic therapy for idiopathic choroidal neovascularization. Am J Ophthalmol 2013;155:713-19.

8 Inoue $\mathrm{M}$, Kadonosono $\mathrm{K}$, Watanabe $\mathrm{Y}$, et al. Results of 1-year follow-up examinations after intravitreal bevacizumab administration for idiopathic choroidal neovascularization. Retina 2010;30:733-8.

9 Hayreh SS. Segmental nature of the choroidal vasculature. $\mathrm{Br} J$ Ophthalmol 1975;59:631-48.

10 Hayreh SS. In vivo choroidal circulation and its watershed zones. Eye 1990; 4(Pt 2):273-89.

11 Giuffre G. Main posterior watershed zone of the choroid. Variations of its position in normal subjects. Doc Ophthalmol 1989;72:175-80.

12 Hayashi K, de Laey JJ. Indocyanine green angiography of submacular choroidal vessels in the human eye. Ophthalmologica 1985;190:20-9.

13 Mendrinos E, Pournaras CJ. Topographic variation of the choroidal watershed zone and its relationship to neovascularization in patients with age-related macular degeneration. Acta Ophthalmol 2009;87:290-6.

14 Hayashi K, de Laey JJ. Indocyanine green angiography of choroidal neovascular membranes. Ophthalmologica 1985;190:30-9.

15 Ross RD, Barofsky JM, Cohen G, et al. Presumed macular choroidal watershed vascular filling, choroidal neovascularization, and systemic vascular disease in patients with age-related macular degeneration. Am J Ophthalmol $1998 ; 125: 71-80$
16 Macular Photocoagulation Study Group. Argon laser photocoagulation for senile macular degeneration: results of a randomized clinical trial. Arch Ophthalmol 1982;100:912-18.

17 Hayreh SS. Submacular choroidal vascular pattern. Experimental fluorescein fundus angiographic studies. Graefes Arch Clin Exp Ophthalmol 1974;192:181-96.

18 Hayreh SS. The choriocapillaris. Graefes Arch Clin Exp Ophthalmol 1974;192:165-79.

19 Shimizu K, Ujiie K. Morphology of the submacular choroid: vascular structure. Ophthalmologica 1981;183:5-10.

20 Hirata Y, Nishiwaki H, Miura S, et al. Analysis of choriocapillaris flow patterns by continuous laser-targetd angiography in monkeys. Invest Ophthalmol Vis Sci 2004;45:1954-62.

21 Hayreh SS, Baines JA. Occlusion of the posterior ciliary artery. 3. Effects on the optic nerve head. Br J Ophthalmol 1972;56:754-64.

22 Hayreh SS, Baines JA. Occlusion of the posterior ciliary artery. II. Chorio-retinal lesions. Br J Ophthalmol 1972;56:736-53.

23 Hayreh SS. Posterior ciliary artery circulation in health and disease: the Weisenfeld lecture. Invest Ophthalmol Vis Sci 2004;45:749-57;748.

24 Giovannini A, Mariotti C, Ripa E, et al. Choroidal filling in age-related macular degeneration: indocyanine green angiographic findings. Ophthalmologica 1994;208:185-91.

25 Tong JP, Chan WM, Liu DT, et al. Aqueous humor levels of vascular endothelial growth factor and pigment epithelium-derived factor in polypoidal choroidal vasculopathy and choroidal neovascularization. Am J Ophthalmol 2006;141:456-62.

26 Spaide RF. Age-related choroidal atrophy. Am J Ophthalmol 2009;147:801-10.

27 Chung SE, Kang SW, Lee JH, et al. Choroidal thickness in polypoidal choroidal vasculopathy and exudative age-related macular degeneration. Ophthalmology 2011;118:840-5.

28 Xu W, Grunwald JE, Metelitsina TI, et al. Association of risk factors for choroidal neovascularization in age-related macular degeneration with decreased foveolar choroidal circulation. Am J Ophthalmol 2010;150:40-7.

29 Gewaily DY, Grunwald JE, Pistilli M, et al. Delayed patchy choroidal filling in the Comparison of Age-Related Macular Degeneration Treatments Trials (CATT). Am J Ophthalmol 2014;158:525-31.

30 Ahn SJ, Kim TW, Ahn J, et al. Subfoveal choroidal thickness in idiopathic choroidal neovascularization. Ophthalmology 2014:34;1554-9. 